\title{
Strates
}

STRATES Matériaux pour la recherche en sciences sociales

$12 \mid 2006$

Nouvelles tensions impériales et recompositions en Europe centrale, orientale, et CEI

\section{La question de la modernisation agricole dans les pays d'Europe centrale}

Perspectives de recherche

\section{Michel STREITH}

\section{OpenEdition \\ Journals}

\section{Édition électronique}

URL : http://journals.openedition.org/strates/2092

DOI : $10.4000 /$ strates.2092

ISSN : $1777-5442$

\section{Éditeur}

Laboratoire Ladyss

Édition imprimée

Date de publication : 31 décembre 2006

ISSN : 0768-8067

\section{Référence électronique}

Michel STREITH, « La question de la modernisation agricole dans les pays d'Europe centrale », Strates [En ligne], 12 | 2006, mis en ligne le 20 juillet 2007, consulté le 08 septembre 2020. URL : http:// journals.openedition.org/strates/2092 ; DOI : https://doi.org/10.4000/strates.2092

Ce document a été généré automatiquement le 8 septembre 2020

Tous droits réservés 


\title{
La question de la modernisation agricole dans les pays d'Europe centrale
}

\author{
Perspectives de recherche
}

\section{Michel STREITH}

1 La question de la modernisation agricole est au cœur du processus d'intégration des pays d'Europe centrale à l'Union européenne (UE). En effet, si les restructurations consécutives à l'effondrement des régimes socialistes semblent irréversibles dans les domaines de l'industrie et des services, la situation est plus complexe dans le secteur agricole. Les changements sociaux en cours ou à venir ont un impact équivalent à celui de la chute du mur de Berlin. Les transformations touchent des millions d'acteurs. La modernisation des exploitations se heurte à une " hérédité sociologique " particulière à ces pays, qui bouleverse les résultats escomptés. En raison des montages financiers considérables, les spécialistes économiques, agronomiques et politistes se livrent à des études prospectives et mobilisent des recherches appliquées. Mais, dans les pays occidentaux, à de très rares exceptions près, les sciences humaines sont absentes des débats liés aux questions agraires en Europe centrale.

2 Les sociétés rurales est-européennes, et plus particulièrement celles de Pologne, de Tchéquie et de Hongrie, relèvent des défis politiques et économiques majeurs comme la transition, la modernisation et l'intégration. Chacune des transformations, menée simultanément, implique une remise en cause des façons de vivre et de penser des populations concernées. De plus, et c'est là l'intérêt de l'approche socioanthropologique, le sens donné à ces termes par les élites politiques nationales ou européennes diffère de la réalité vécue par les acteurs.

La transition

3 Ainsi, la transition est essentiellement conçue, du point de vue des dirigeants, comme une «fiction de la réversibilité de l'histoire » (Giordano, 1994). Depuis plus de dix ans, tout est mis en œuvre pour effacer l'expérience socialiste. On peut parler d'une véritable «congélation de l'histoire». Certes, la mise entre parenthèses du passé 
socialiste correspond à une conception de la transition comme période, c'est-à-dire comme temps nécessaire pour passer d'un état à un autre, pour revenir à un ordre «normal » ou « naturel » des choses. Un tel paradigme a servi de support aux politiques économiques de la transition, aux "thérapies de choc" (Dontaine, 1993). Il renvoie également à la théorie de la «fin de l'histoire». Mais, la définition n'est pas univoque. La transition peut être caractérisée comme un état particulier susceptible d'être théorisé ou un processus dont les composantes restent à déterminer. L'approche en termes de période suppose une coupure radicale avec l'ancien système ; les deux autres reconnaissent des éléments de continuité, de permanence. Ainsi pouvons-nous analyser, dans cette perspective, le phénomène de "repli paysan " (Maurel, 2003), caractéristique imprévue, mais repérable d'un point de vue diachronique, des changements dans les campagnes est-européennes.

La modernisation

4 À ce flou conceptuel qui enrobe la transition s'ajoute celui de la modernisation. Les deux questions semblent liées. La modernisation serait, en quelque sorte, le parachèvement, en termes de production, de la politique de transition. Or, nous l'avons souligné, la transition n'est pas conçue comme une recherche d'avancées sociales ou économiques mais comme un retour à une situation ante socialiste. De plus, la période socialiste fut elle-même définie en son temps, par ses dirigeants, comme un dépassement des anciennes structures archaïques du capitalisme. Dès lors, il devient délicat de définir le type de modernisation dont il est question aujourd'hui tant les temporalités se renversent ou se superposent. Le terme n'échappe pas, lui non plus, à des effets de contexte et à des manipulations idéologiques.

5 Henri Mendras a défini quatre formes particulières de modernisation agricole (Mendras, 1984). Une première, dite globale consiste à "apporter des solutions concrètes et progressives aux problèmes posés par la nécessité du changement ». C'est la version couramment admise par les élites politiques dans les pays en voie de transition. Sa portée théorique en sciences humaines est limitée. Tout d'abord, elle induit une conception déterministe et monolythique des transformations sociales. Les changements sont inéluctables et impulsés «du haut». Les acteurs sont exclus des processus de décision et leurs tentatives d'imposer une alternative participent de ce que les sociologues du modernisme appellent des "destructions créatrices" (Zapf, 1994). Ensuite, l'approche globalisante du phénomène écarte toute analyse en profondeur des adaptations régionales ou locales à la modernisation.

6 Une deuxième forme, dite symbolique, joue sur les contradictions d'un système. Elle combine des éléments techniques nouveaux et une réutilisation d'anciens procédés. H. Mendras cite l'exemple du tracteur acheté pour tirer la charrette autrefois attelée aux chevaux. Le ressort de cette modernisation est l'imitation sociale: il convient d'être possesseur de matériels ou de produits récents en les détournant de leurs usages, juste pour «faire comme le voisin». Cette pratique est néanmoins limitée par le coût d'investissement. En effet, l'adoption de nouvelles techniques implique des financements qui ne sont pas toujours compatibles avec les revenus de l'agriculteur.

7 Une troisième forme, dite traditionnelle, vise à adapter les trois éléments constitutifs de la ferme familiale, le sol, le travail et le capital, aux nouvelles donnes économiques. Cela rejoint la conception de l'économie paysanne élaborée par Alexandre Chayanov. La dimension de la famille constitue le critère d'évaluation du développement de la ferme (Chayanov, 1966). Lors d'une augmentation des prélèvements extérieurs ou en cas 
d'impossibilité d'accroître les superficies, les membres familiaux travaillent davantage. Aujourd'hui, ce supplément de tâches s'effectue sur l'exploitation même, dans le cadre du développement des activités touristiques par exemple, ou, plus fréquemment, à l'extérieur, dans le cadre du travail saisonnier ou de la double activité.

Enfin, une quatrième forme de modernisation, dite progressiste, consiste à conserver les structures de l'exploitation (surfaces, matériel, forces de travail) tout en transformant radicalement le système de production et de gestion. C'est une version plus «politique» que la précédente. Elle prend aujourd'hui la forme d'une spécialisation dans des secteurs à forte valeur ajoutée. C'est ce que les spécialistes commerciaux appellent des «niches » telles l'agriculture biologique ou les productions spécialisées (la groseille ou la framboise en Pologne, le Tokaj ou le miel en Hongrie par exemple).

\section{L'intégration}

9 Les problèmes théoriques soulevés par la transition et la modernisation, ou les modernisations, nous amènent à reconsidérer la question plus générale de l'intégration de nouveaux pays d'Europe centrale à l'UE. En effet, la transition a consisté, dans un premier temps, à transférer le modèle de l'exploitation agricole ouest-occidentale. Cependant, les mesures adoptées se sont heurtées à une série de contradictions. La rapidité des privatisations, la discrimination entre ceux qui possèdent la terre et ceux qui la travaillent et l'insuffisance des aides à l'installation ont produit des effets contraires à ceux initialement escomptés. Certes, des exploitations sont devenues aussi compétitives que celles de l'Ouest. Mais, elles représentent une infime minorité des fermes de Pologne, de Tchéquie et de Hongrie. De plus, elles bénéficient de soutiens financiers disproportionnés et sont sous la menace d'une réorientation des subventions européennes. En fait, la majorité des exploitations en activité sont de petite taille et proche du modèle paysan courant en Europe centrale depuis des décennies. Une étude approfondie, coordonnée par le Centre d'Études et de Recherches Internationales, visant à préparer l'intégration de la Pologne dans l'UE, nous fournit des données intéressantes. Aujourd'hui, 2,8 millions d'agriculteurs polonais vivent dans des exploitations de moins de 7 ha. Ces petites structures représentent $87 \%$ du total des exploitations agricoles de ce pays (Bafoil, 2003).

Les micro- et petites exploitations limitent à elles seules un taux de chômage déjà très élevé : $17 \%$. Elles sont même le rempart contre une catastrophe sociale dans les campagnes. Elles assurent un faible revenu, voire permettent de survivre. Les spécialistes agronomiques les qualifient, avec beaucoup de condescendance, d'exploitations «sociales ». Il suffirait pourtant d'aides financières ou d'un agrandissement des surfaces pour qu'elles assurent un revenu correct. C'est d'ailleurs le cas de celles qui se sont spécialisées et cultivent entre 5 et 7 ha. Ainsi, la Pologne estelle parmi les trois premiers producteurs mondiaux, en volume, de groseilles et de framboises. De très bons résultats s'observent également dans la production de choux, de fraises ou de pommes. Cette faculté d'adaptation tient, en grande partie, à l'évolution de l'agriculture depuis la fin $\mathrm{du}$ xix ${ }^{\mathrm{e}}$ siècle. Au fil des décennies, les phénomènes de double activité et d'adaptabilité à la demande se sont amplifiés. Aujourd'hui, se pose le problème du subventionement. Ces exploitations ne bénéficient d'aucune aide. Néanmoins, un projet national est à l'étude. Dans le cadre d'un plan de développement rural, entre 200000 et 300000 petits exploitants vont bénéficier d'une aide annuelle comprise entre 750 et 1000 euros par an. Le montant est dérisoire au 
regard des subventions et des facilités de crédit offertes aux entreprises de grande taille. Néanmoins, le gouvernement polonais semble obligé de prendre en compte l'importance du fait social dans les campagnes. En effet, la stratégie politique adoptée jusqu'ici se limitait à régler les négociations d'adhésion, c'est-à-dire à pérenniser les exploitations compétitives. Or, cela ne résout en rien le problème de suremploi massif dans le secteur agricole. À l'héritage socialiste qui a "produit " près de $20 \%$ d'actifs agricoles, se surajoute le phénomène de « repli sur la terre ». De nombreux laissés pour compte des privatisations industrielles, retraités, chômeurs, ont réinvesti leurs parcelles pour en tirer de quoi survivre.

L'héritage sociologique

11 Le problème démographique et celui de l'héritage sociologique constituent les deux points centraux de la problématique de l'intégration de la Pologne, de la Tchéquie et de la Hongrie. Dans ces trois pays, le nombre de ruraux est pratiquement resté identique depuis près d'un siècle, contrairement aux pays d'Europe occidentale. L'industrialisation s'est effectuée dans des bassins très limités et les pôles urbains se sont développés plus lentement et de manière moins centralisée. Dès lors, de nombreuses régions ont conservé une population essentiellement tournée vers l'activité agricole. Dès les années 1930, un phénomène de surpopulation rurale est à l'œuvre et les politiques agricoles visent à contenir le mécontentement dans les campagnes et à faciliter l'accès à la terre pour les petits agriculteurs. Au-delà de leur aspect populiste et politique, les mouvements agrariens, par exemple, peuvent être analysés économiquement sous cet angle. George Jackson rappelle qu'à cette époque, les pays d'Europe centrale sont en situation de pays colonisés: les rares capitaux investis proviennent de pays étrangers, les productions sont destinées à l'exportation et l'industrialisation y est faible. L'auteur souligne que l'industrialisation ne relève pas uniquement d'un "héritage historique». Certes, le servage subsiste en bien des régions. L'aristocratie foncière détient un pouvoir politique fort et n'insuffle pas les capitaux nécessaires au développement local. Mais, la domination économique de l'Europe centrale par les capitalistes occidentaux joue un rôle non négligeable. Les investissements étrangers ne permettent pas un développement industriel de ces régions. D'ailleurs, lorsqu'ils existent, ils sont nettement insuffisants. À l'inverse des pays occidentaux, l'agriculture ne bénéficie pas des «retours » de l'accroissement du potentiel industriel (utilisation des engrais chimiques, transformation des produits, développement d'un marché intérieur). Les mouvements agrariens poussent sur ce terreau. Pour leurs représentants, il s'agit de s'en sortir en impulsant une modernisation agricole spécifique, indépendante de l'industrialisation et des capitaux occidentaux. En ce sens, les mouvements agrariens, selon le cliché entretenu, sont moins porteurs d'une idéologie paysanne conservatrice que soucieux d'adapter l'agriculture à une situation politique et économique défavorable. Cela a expliqué, en partie, leur succès auprès des populations rurales (Jackson, 1974).

$12 \mathrm{Au}$ lendemain de la Seconde Guerre mondiale, les autorités communistes font des réformes agraires l'événement fondateur de l'instauration des nouveaux régimes. Certes, les mises en application varient d'un pays à l'autre. Mais, au-delà de l'objectif politique visant à promouvoir l'alliance des ouvriers et des paysans, ces réformes participent au maintien d'actifs agricoles. J'ai démontré dans ma thèse, à partir de l'exemple du Mecklembourg, en ex-RDA, comment les quarante années de politique agricole du régime communiste obéissaient tout autant à des présupposés idéologiques qu'à la volonté de régler un problème démographique né de l'afflux massif de 
populations nouvelles dans les campagnes. Concernant la situation actuelle, l'on peut avancer l'hypothèse suivante : les autorités se doivent d'orienter leur politique agricole en fonction de ce soubassement démographique hérité de périodes antérieures. Dans ce domaine, les pouvoirs en place depuis la fin du communisme "naviguent à vue ", privilégiant les critères d'adéquation avec la Politique agricole commune (PAC) plutôt qu'une vision à long terme. Mais le problème demeure. C'est en ce sens que MarieClaude Maurel, Maria Halamska et Hugues Lamarche emploient le concept de «modernisation différée» pour analyser le processus de changement dans l'agriculture. Le problème est récurrent en Europe centrale. En effet, dans des contextes historiques différents, l'on assiste, depuis plusieurs décennies, à des «modernisations inachevées» (Streith, 2005). Ainsi, les réformes agraires successivement mises en place n'ont jamais pu bénéficier d'une périodicité nécessaire à leur consolidation. Les bouleversements politiques (guerres, changements de régime) ou économiques (collectivisation des moyens de production par exemple) ont fréquemment stoppé les dynamiques de développement des fermes familiales.

Le deuxième point central de la problématique de l'intégration tient à la question sociale. Contrairement aux politiques de modernisation menées dans les pays d'Europe occidentale, par exemple celle de 1960 en France, il est impossible de séparer l'agricole du rural en Europe centrale. Plus précisément, l'on ne peut pas tenter de régler la question de la production ou de la productivité sans tenir compte de celle du social. L'histoire de l'Europe occidentale nous enseigne que les politiques de modernisation agricole ont privilégié, dans un premier temps, l'augmentation de la productivité ou de la compétitivité. Elle nous incite à prendre en compte, dans un deuxième temps, les conséquences sociales de ces choix. Cela explique, en grande partie, le phénomène d'exode. Ce processus n'est guère concevable en Europe centrale où l'industrie et les services n'offrent pas d'alternative au travail agricole en milieu rural. Tout projet de développement agricole devra prendre en compte simultanément l'économique et le social.

L'échelle d'analyse

Pour tenter de combler les lacunes théoriques énoncées précédemment, il est nécessaire d'adopter une méthodologie privilégiant trois champs disciplinaires complémentaires. Tout d'abord, d'un point de vue ethnologique, il convient d'effectuer une analyse fine et qualitative de l'exploitation agricole. En effet, au cœur des expériences passées, des comportements actuels et des "horizons d'attente" concernant le problème de la modernisation agricole se trouve la question des savoirs techniques et symboliques élaborés au sein de l'espace domestique. Plus fondamentalement, l'exploitation agricole est à l'interface du local et du global. Elle permet de saisir les liens complexes entre la sphère privée (les liens de parenté, les réseaux, la transmission des savoirs) et la sphère publique (le contexte régional, national, et la globalisation), à travers notamment la répartition des aides européennes. Ces dernières sont encore principalement attribuées en direction du secteur agraire. Pour explorer ces liens, il convient d'analyser une variété d'exploitations réparties sur l'ensemble des territoires des pays concernés. L'exploitation agricole comme objet d'étude autorise l'analyse conjointe des trajets de vie, des transformations sociales locales et de l'évolution des modes et techniques de production. Pour cela, plusieurs sources sont nécessaires: les histoires de vie, les archives locales et régionales, la littérature grise et les statistiques. Cette approche à multiples facettes, par incrustation, prenant en compte des données qualitatives et quantitatives, nous permet 
de comprendre comment « les systèmes travaillent réellement » et de saisir dans quelle mesure « le pays réel s'oppose au pays légal » (Hann, 1993).

Le travail ethnographique est complété par une approche historique. La mise en relation des histoires de vie et du déroulement chronologique fait apparaître de profonds antagonismes sociaux. Les récits oraux construisent généralement l'histoire locale, régionale et nationale comme une succession de conflits. C'est une donnée fréquente dans les pays sortant du communisme. Différentes logiques sont à l'œuvre : le rapport à la propriété et à l'usage de la terre, les mouvements de population et/ou les bouleversements politiques. La succession de pouvoirs contradictoires dans ces régions depuis trois générations brouille le statut social des individus. Un réexamen de l'histoire agraire contemporaine, dans une perspective diachronique, est donc indispensable. Il convient de tenir compte d'un double déroulement du temps : d'une part, celui de la chronologie fine établie par les historiens et s'appuyant sur des événements politiques ou économiques précis, et, d'autre part, celui des scansions spécifiques au monde paysan. Cela nous permet de comprendre les décalages et distorsions observables aujourd'hui.

L'approche de la problématique paysanne en termes d'économie politique constitue le troisième volet méthodologique. Les politiques agricoles, successivement mises en place au gré des changements de régime, obéissent à des temporalités opposées. L'une est syntagmatique, indépendante des modifications historiques ou géographiques. Il s'agit du rôle général de l'agriculture dans une société, de ses " soucis divergents » pour reprendre l'expression d'H. Mendras. L'autre est paradigmatique. Elle relève de choix opérés dans des situations historiques successives et des substitutions de modèles les accompagnant. Toute politique agricole est donc discriminatoire. Elle privilégie un modèle et, par voie de conséquence, des acteurs qui la mettent en œuvre. Les autres s'adaptent, élaborent des stratégies alternatives ou disparaissent. Ce phénomène s'observe avec netteté dans les sociétés postsocialistes.

La méthodologie adoptée renvoie à une approche de type "socio-anthropologique ». Il faut entendre par là l'étude empirique d'un groupe social, les agriculteurs, combinant une analyse des pratiques et celle des représentations. La sociologie nous fournit les instruments pour une mise en perspective diachronique des phénomènes. L'anthropologie privilégie les histoires de vie et autorise l'approche comparatiste de groupes sociaux resitués dans un territoire donné. En ce sens, le parti-pris adopté consiste à aborder le processus de modernisation agricole dans sa dimension dynamique. Nous pouvons illustrer ce choix à partir de la question de la typologie des exploitations.

La question des typologies

18 Les critères d'évaluation de l'entrée de nouveaux pays dans l'UE sont élaborés, dans le domaine agricole, à partir d'une typologie des exploitations. Celle-ci détermine le choix des politiques agricoles nationales et de la répartition des aides communautaires. L'enjeu est donc considérable tant du point de vue financier que du point de vue du devenir même de l'agriculture. Cependant, les choix méthodologiques qui président à leur constitution posent problème. Ainsi, dans le cas de la Pologne, qui a fait l'objet d'un «traitement particulier» en raison de la complexité de la question agraire, les critères retenus sont les résultats économiques des exploitations. Deux seuils sont retenus : l'un, dit de survie, est le revenu nécessaire aux besoins vitaux du ménage et au maintien de l'exploitation en l'état; l'autre, dit de renouvellement, permet à 
l'agriculteur de tirer de son travail un revenu suffisant et d'accroitre le potentiel de sa ferme (achat de matériel et d'intrants). La typologie est ensuite déclinée à partir de ces deux paramètres: micro-exploitation, petite exploitation, moyenne exploitation, grande exploitation et exploitation patronale.

19 Cette typologie économiste et agronomique est réductrice. Tout d'abord, géographiquement, elle part du postulat d'un espace homogène et ne tient pas compte des recompositions territoriales. Ainsi, une petite exploitation proche d'un centre urbain n'a pas la même valeur économique que celle située dans une région périphérique. Les possibilités de double activité pour l'agriculteur ou l'existence de débouchés pour les productions varient considérablement en fonction de la localisation de l'exploitation. Certes, le revenu de l'exploitant peut être calculé dans un temps et un espace donnés. Mais, son évolution est susceptible de modifications rapides, dans un sens positif ou négatif, au regard des politiques régionales ou locales de développement. Ensuite, historiquement, l'on ne peut pas traiter de manière uniforme des lieux à héritage différent. Parler de petite exploitation en Poméranie ou en Mazurie ne signifie pas la même chose. L'une s'est constituée à l'ombre des grands domaines, l'autre est issue de la concurrence foncière entre groupes sociaux antagonistes. Leur existence et leur pérennisation tient à leur appartenance ou non à un système agraire complexe. Enfin, sociologiquement, la typologie économiste sous-estime les mécanismes de représentation des acteurs. Nous avons rappelé, à partir de la classification d'H. Mendras, les multiples facettes de la modernisation. Les choix opérés par les agriculteurs tiennent autant à des facteurs économiques objectifs qu'à des systèmes de pensée adaptables et modulables suivant les situations historiques. Ainsi, le maintien de la micro-exploitation relève-t-il autant d'un souci de survivre dans un contexte économique difficile que de conserver un patrimoine foncier pouvant être éventuellement réutilisé.

Apporter la contradiction à l'approche réductrice de la typologie économiste, c'est rappeler que la réalité de l'exploitation agricole est polymorphe. Comme le souligne Marie-Claude Maurel, il convient de prendre en compte les «différents systèmes qui structurent le fonctionnement de l'exploitation (systèmes de production, systèmes techniques, systèmes familiaux, systèmes économiques, systèmes de valeurs et de représentations, etc.) " (Maurel, 2003). À partir de ce recadrage théorique, l'auteur élabore, dans le cas de la Pologne, trois modèles distincts : le modèle paysan ou vivrier, les modèles entrepreneuriaux et le modèle alternatif ou de transition. Cette typologie, constituée avec des outils conceptuels particuliers aux sciences humaines, autorise des interprétations plus larges de la réalité dans la mesure où elle tient compte des dynamiques économiques, politiques et sociales passées et présentes. Elle déconstruit sans aucun doute les clichés entretenus autour de la ferme compétitive/ferme "sociale» et donne toute son épaisseur sociologique à la réalité des exploitations agricoles en Europe centrale. 


\section{BIBLIOGRAPHIE}

Bafoil F., Guyet R., L'Haridon L., Tardy V., 2003, « Pologne. Profils d'agriculteurs », Le courrier des pays de l'Est, $\mathrm{n}^{\circ} 1034$, p. 28-45.

Chayanov A., 1966, The Theory of Peasant Economy, Richard D. Irwin, Homewood.

Dontaine A., 1993, « Transition en Europe centrale et orientale. Questions théoriques et pratiques », Essais sur le discours de l'Europe éclatée, 11, p. 205-213.

Giordano C., 1994, « Historizität statt Modernisierung ? Reflexionen über transformationsprozesse in Mittel- und Osteuropa », in Brombach C. et Nebelung A. (Hrsg.), Zwischenzeiten und Seitenwege. Lebensverhältnisse in peripheren Regionen, Münster/Berlin, LIT Verlag, p. 217-232.

Hann C. (ed.), 1993, Socialism: Ideals, Ideologies and Local Practice, London/New York, Routledge.

Jackson George D., 1974, « Peasant Political Movements in East Europe 1919-1930 », in Landsberger H. A. (ed.), Rural Protest: Peasant Movements and Social Change, Londres, Macmillan, p. 259-315.

Maurel M.-C., Halamska M., Lamarche H., 2003, Le repli paysan, Paris, L'Harmattan.

Mendras H., 1970, La fin des paysans, Paris, Armand Colin.

Streith M., 2005, Dynamiques paysannes en Mecklembourg. Survie d'un savoir-faire, Münster/Berlin, LIT Verlag.

Zapf W., 1994, Modernisierung, Wohlfartsentwicklung und Transformation: soziologische Aufsätze 1987 bis 1994, Berlin, Sigma.

\section{RÉSUMÉS}

L'article aborde les transformations agricoles en cours en Europe centrale en prenant en compte différentes échelles d'analyse. Tout d'abord, il convient de replacer la question agraire dans le contexte général de la transition des espaces post-communistes et de l'intégration de nouveaux pays à l'Union européenne. Ensuite, le processus de modernisation des structures d'exploitation est examiné à la lueur des expériences occidentales et de leurs transferts éventuels en Europe centrale. Enfin, la notion d'héritage sociologique est sollicitée pour aborder les réalités régionales et locales spécifiques.

Michel Streith. The question of agricultural modernisation in Central Europe : perspectives of research The article stresses the on going agricultural changes in Central Europe, taking into account different scales of analysis. At first, it replaces the agrarian question into the general context of the post-communist transition and of the integration of the new member states into European Union. Then, it mentions the modernisation process of production stuctures to the light of occidental experiences and their potential transfers to Central Europe context. At least, the notion of sociological heritage is requested to analyse the specific and local realities. 
AUTEUR

MICHEL STREITH

Chercheur Ladyss, CNRS, michelstreith@wanadoo.fr 